\title{
Green and Facile Preparation of Readily Dual-Recyclable Thermosetting Polymers with Superior Stability based on Asymmetric Acetal
}

Qiong Li ${ }^{1,2}$, Songqi Ma ${ }^{*}$, Sheng Wang ${ }^{1,2}$, Liu Yanlin ${ }^{1}$, Muhammad Abu Taher ${ }^{1,2}$ Binbo Wang ${ }^{1}$, Kaifeng Huang ${ }^{1}$, Xiwei $\mathrm{Xu}^{1}$, Yingying Han ${ }^{1}$, Jin $\mathrm{Zhu}^{1}$

${ }^{1}$ Key Laboratory of Bio-based Polymeric Materials Technology and Application of Zhejiang Province,

Ningbo Institute of Materials Technology and Engineering, Chinese Academy of Sciences, Ningbo 315201,

P. R. China;

${ }^{2}$ University of Chinese Academy of Sciences, Beijing 100049, P. R. China;

*Corresponding authors: (Songqi Ma) E-mail masongqi@nimte.ac.cn, Tel 86-0574-87619806. 


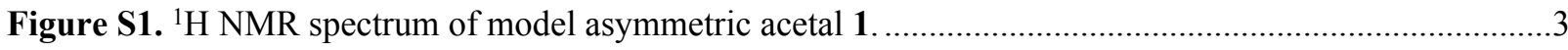

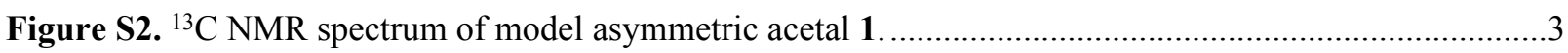

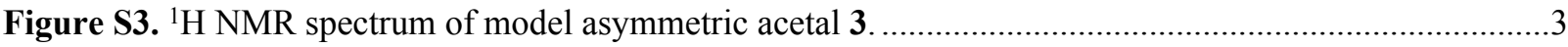

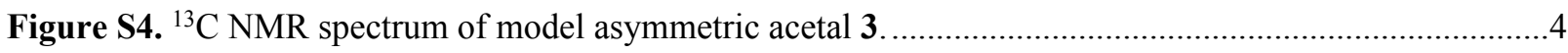

Figure S5. Curing curves for different heating rates of PRC-0.9 system.........................................................

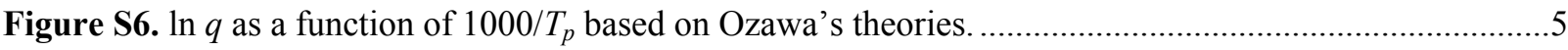

Figure S7. Real-time temperature-dependent FTIR spectra of PRC-0.9 system. .........................................5

Figure S8. Real-time temperature-dependent FTIR spectra of PRC-0.9 system. .........................................6

Figure S9. Real-time temperature-dependent FTIR spectra of -OH in PRC-0.9 system. ................................6

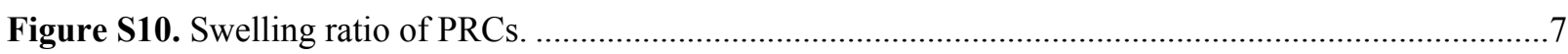

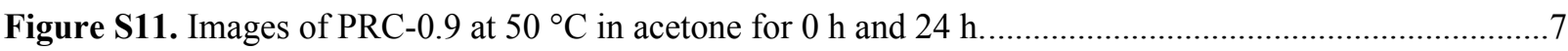

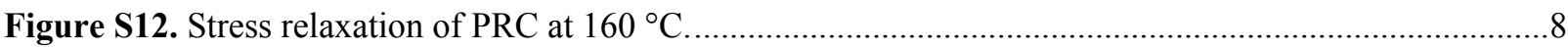

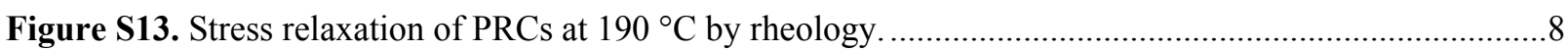

Figure S14. Arrhenius analysis of the characteristic relaxation time $\tau^{*}$ versus $1000 /$ T for PRCs...................9

Figure S15. Representative tensile stress-strain curves of original and reprocessed PRC-0.5 .....................9

Figure S16. Representative tensile stress-strain curves of original and reprocessed PRC- $0.75 \ldots \ldots \ldots \ldots \ldots \ldots \ldots . . .10$

Figure S17. Representative tensile stress-strain curves of original and reprocessed PRC-1.0 ....................10

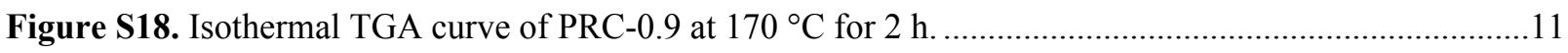

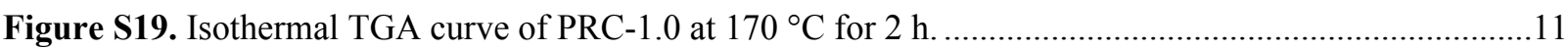

Figure S20. Gas chromatograms of transacetalization between model asymmetric acetal $\mathbf{1}$ and phenol $\mathbf{2}$ at 150 ${ }^{\circ} \mathrm{C}$ for different time.

Figure S21. Gas chromatograms of metathesis between model asymmetric acetals $\mathbf{1}$ and $\mathbf{3}$ at $150{ }^{\circ} \mathrm{C}$ for

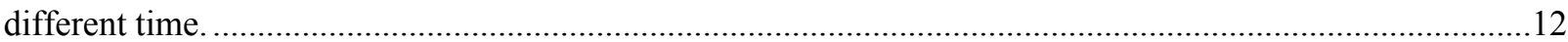

Figure S22. Mass spectrum of model asymmetric acetal 1 separated by gas chromatograph. .......................13

Figure S23. Mass spectrum of model phenol 2 separated by gas chromatograph. ........................................13

Figure S24. Mass spectrum of model asymmetric acetal $\mathbf{3}$ separated by gas chromatograph. ........................14

Figure S25. Mass spectrum of model phenol 4 separated by gas chromatograph. .........................................14

Figure S26. Mass spectrum of model asymmetric acetal 5 separated by gas chromatograph. .......................15

Figure S27. Mass spectrum of model asymmetric acetal $\mathbf{6}$ separated by gas chromatograph. .......................15

Figure S28. Pseudo-first order kinetics of transacetalization.......................................................................16

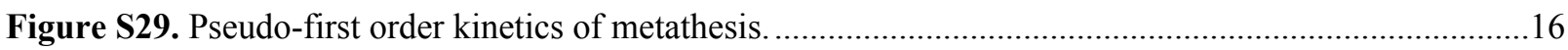

Figure S30. Contact angle of different solvents (water/acetone (v/v)) on PRC-0.9. ......................................17

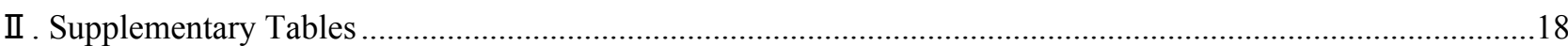

Table S1. The kinetic parameters of the different curing systems. ...........................................................18

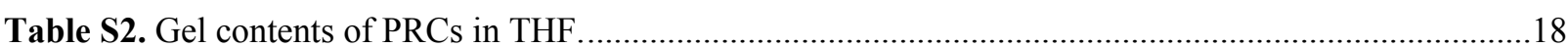

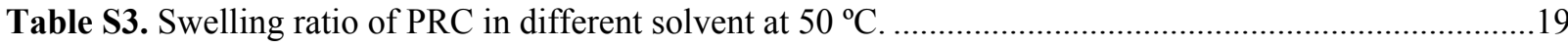

Table S4. Mechanical properties of the original and reprocessed PRCs......................................................20

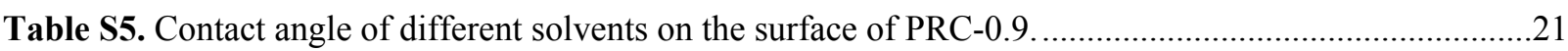

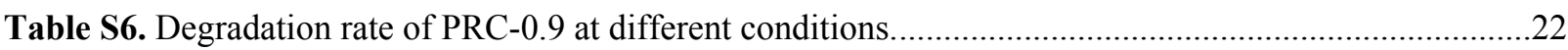

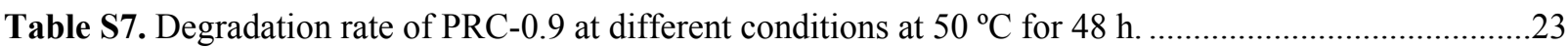




\section{I . Supplementary Figures}

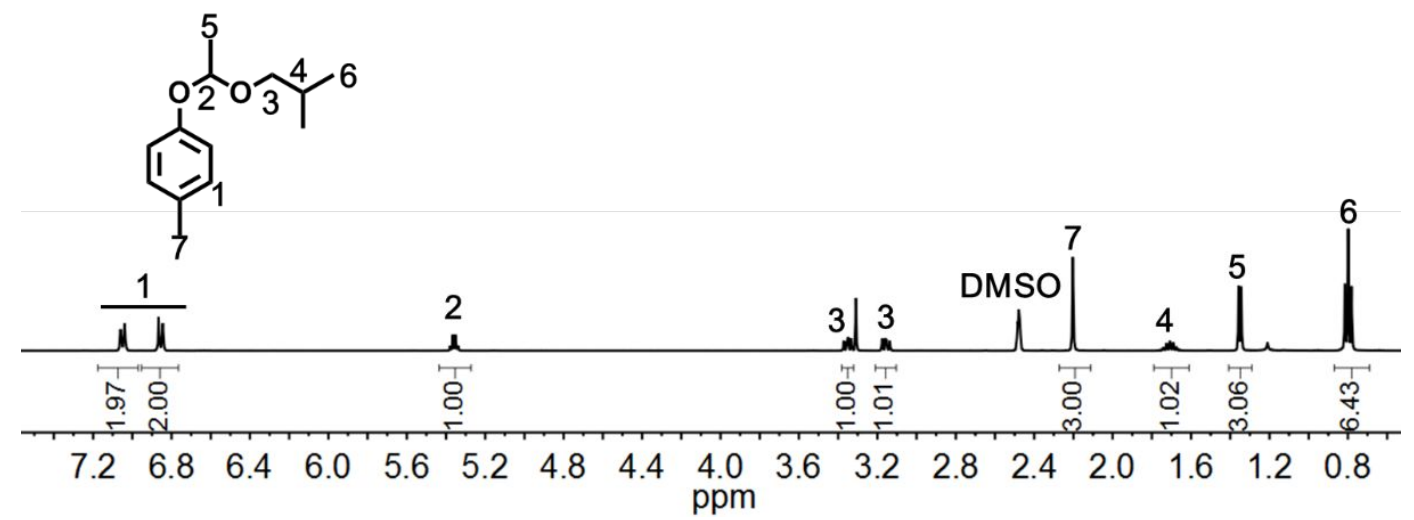

Figure S1. ${ }^{1} \mathrm{H}$ NMR spectrum of model asymmetric acetal 1.

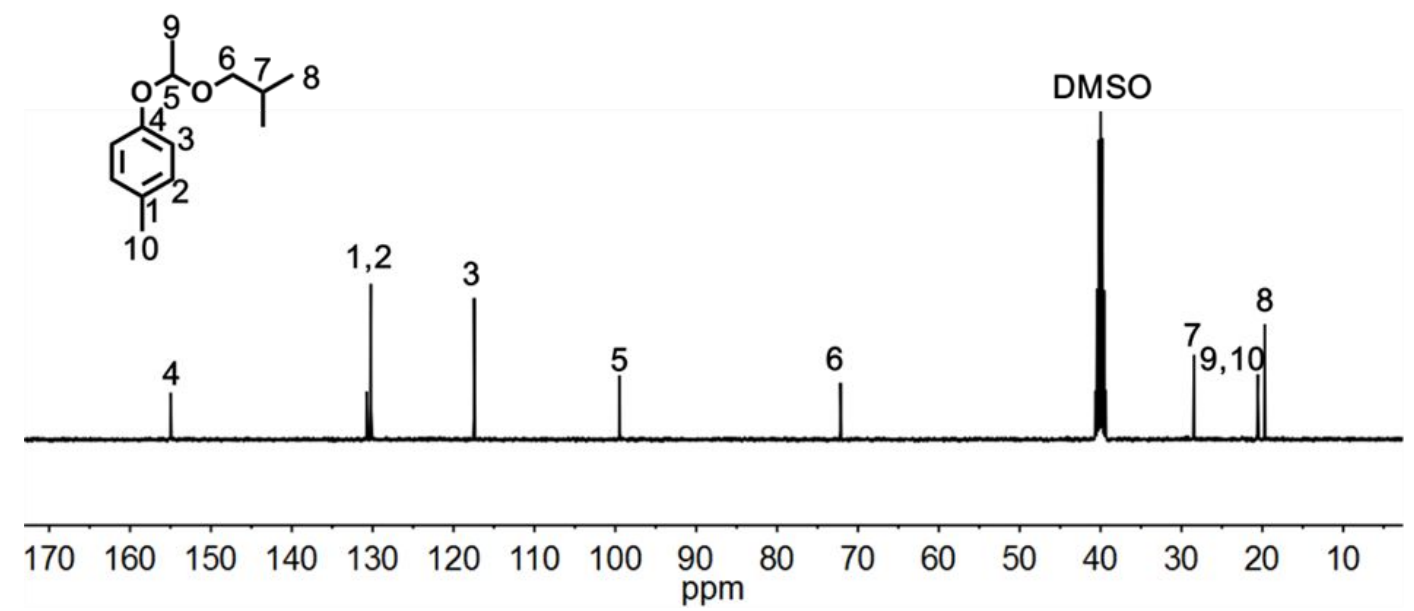

Figure S2. ${ }^{13} \mathrm{C}$ NMR spectrum of model asymmetric acetal $\mathbf{1}$.

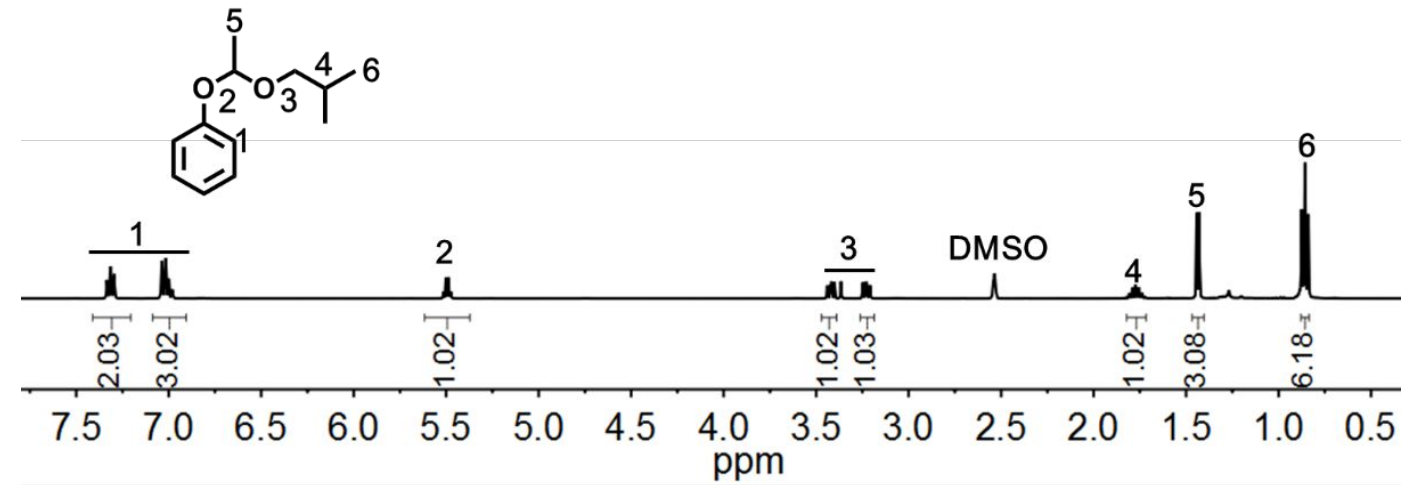

Figure S3. ${ }^{1} \mathrm{H}$ NMR spectrum of model asymmetric acetal 3. 


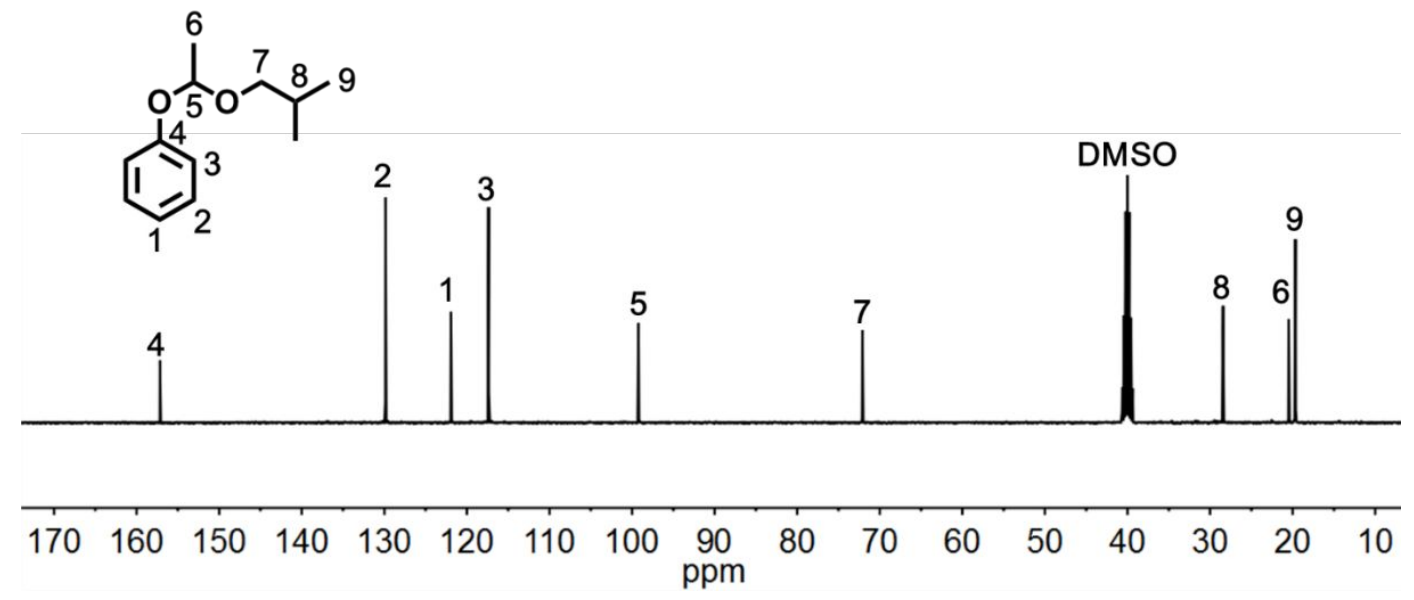

Figure S4. ${ }^{13} \mathrm{C}$ NMR spectrum of model asymmetric acetal 3.

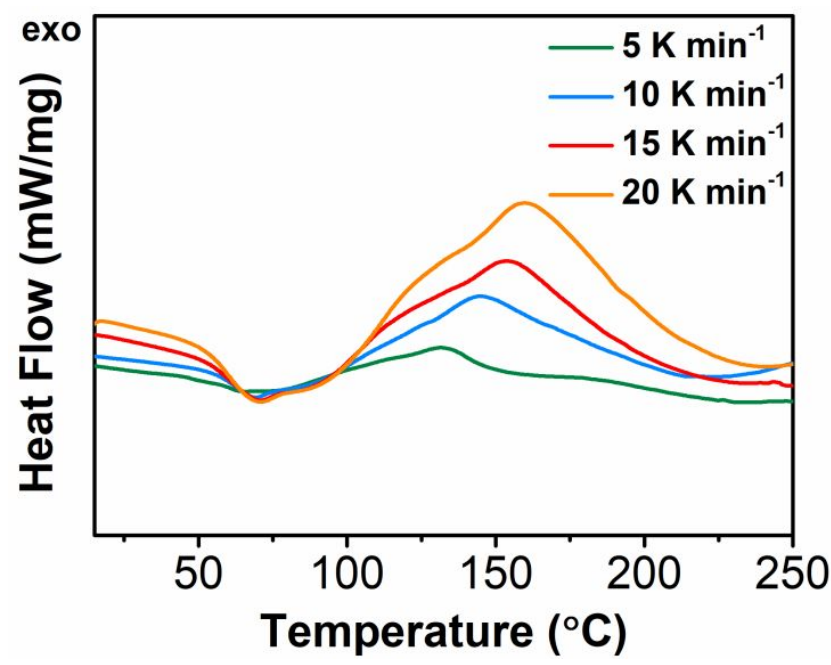

Figure S5. Curing curves for different heating rates of PRC-0.9 system. 


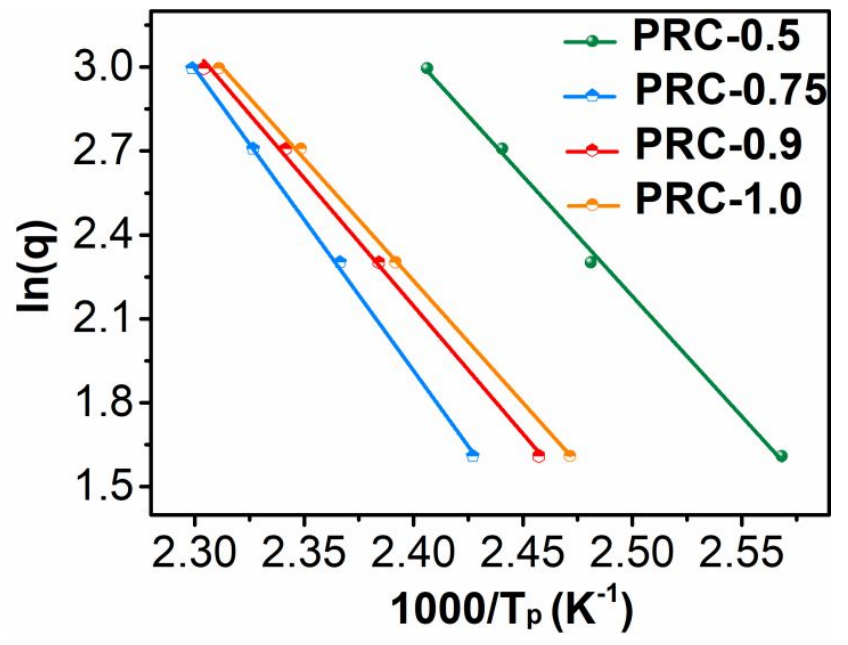

Figure S6. $\ln q$ as a function of $1000 / T_{p}$ based on Ozawa's theories.

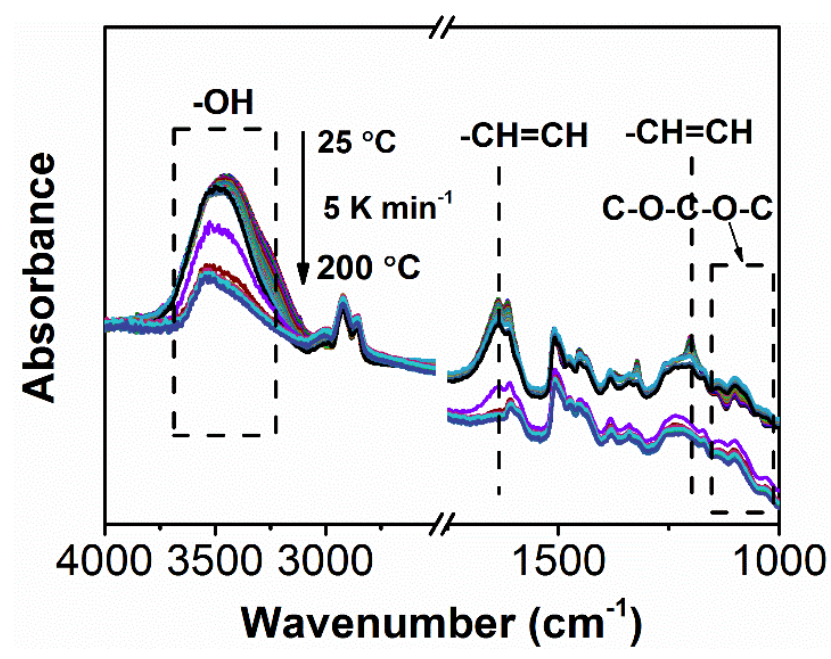

Figure S7. Real-time temperature-dependent FTIR spectra of PRC-0.9 system. 


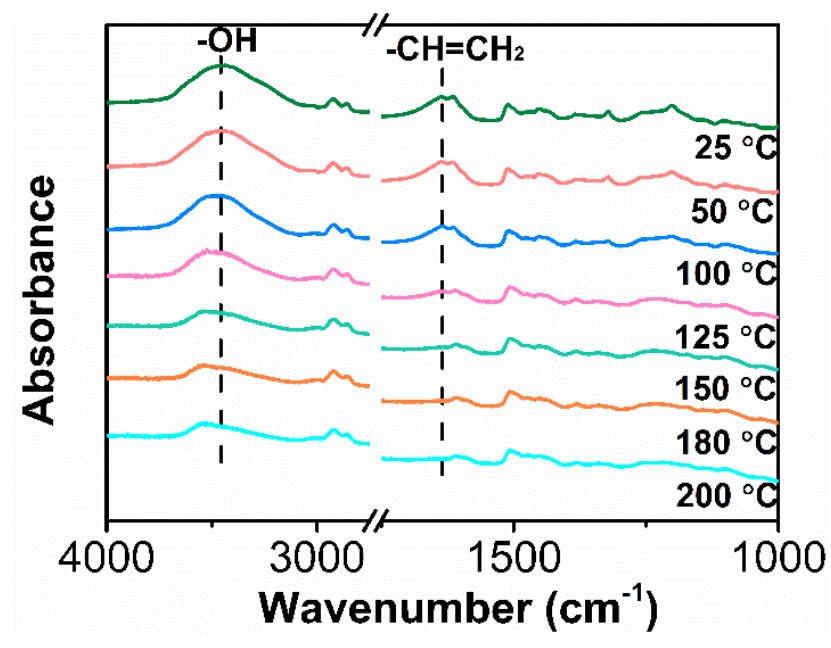

Figure S8. Real-time temperature-dependent FTIR spectra of PRC-0.9 system.

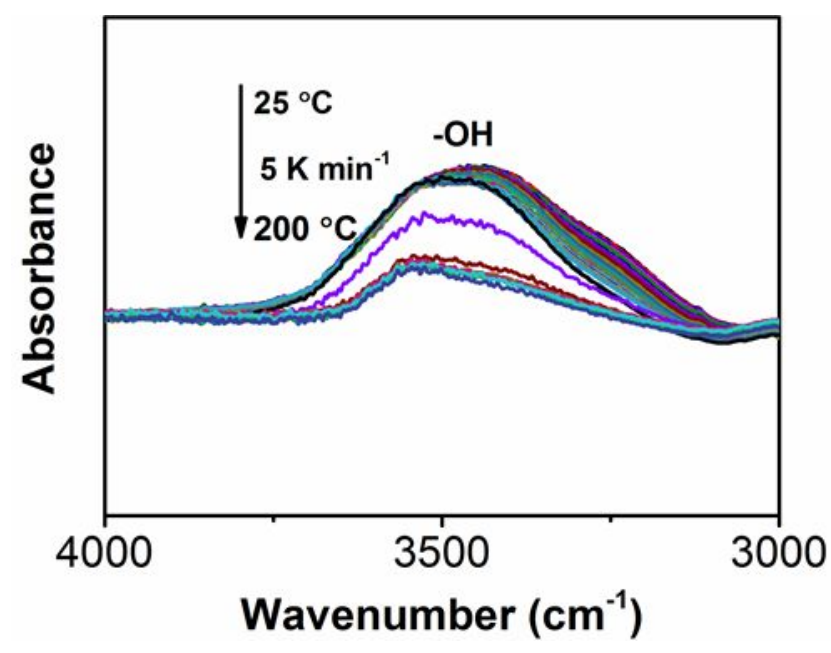

Figure S9. Real-time temperature-dependent FTIR spectra of -OH in PRC-0.9 system. 


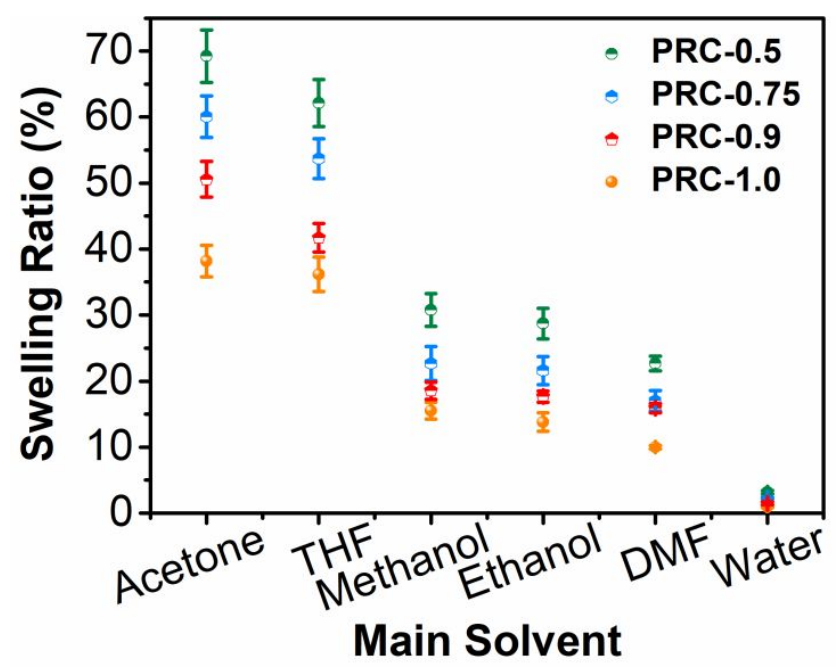

Figure S10. Swelling ratio of PRCs.

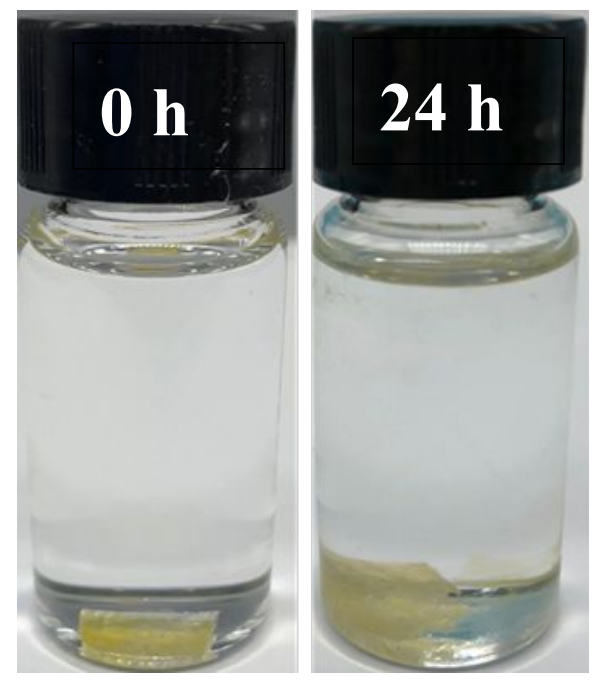

Figure S11. Images of PRC-0.9 at $50{ }^{\circ} \mathrm{C}$ in acetone for $0 \mathrm{~h}$ and $24 \mathrm{~h}$. 


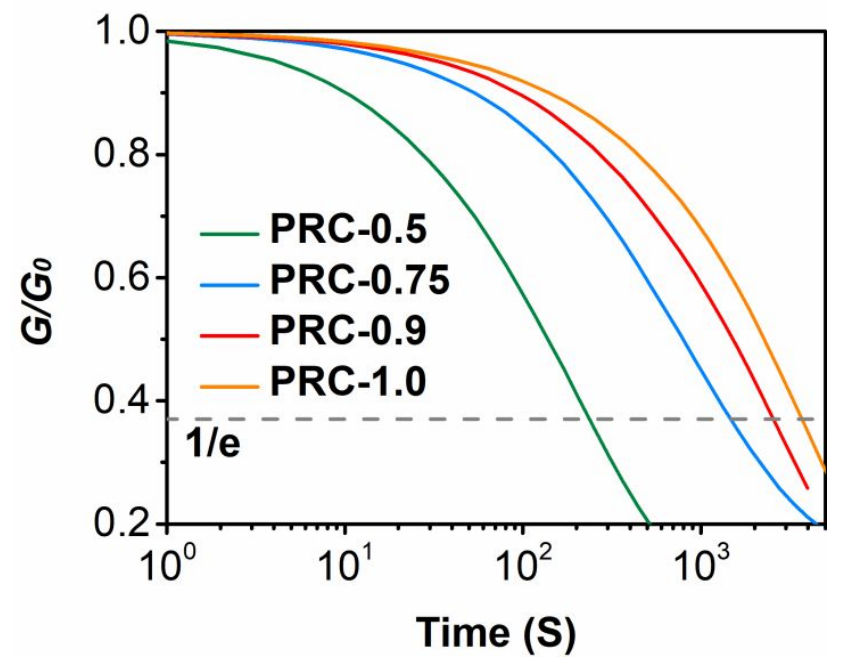

Figure S12. Stress relaxation of PRC at $160^{\circ} \mathrm{C}$.

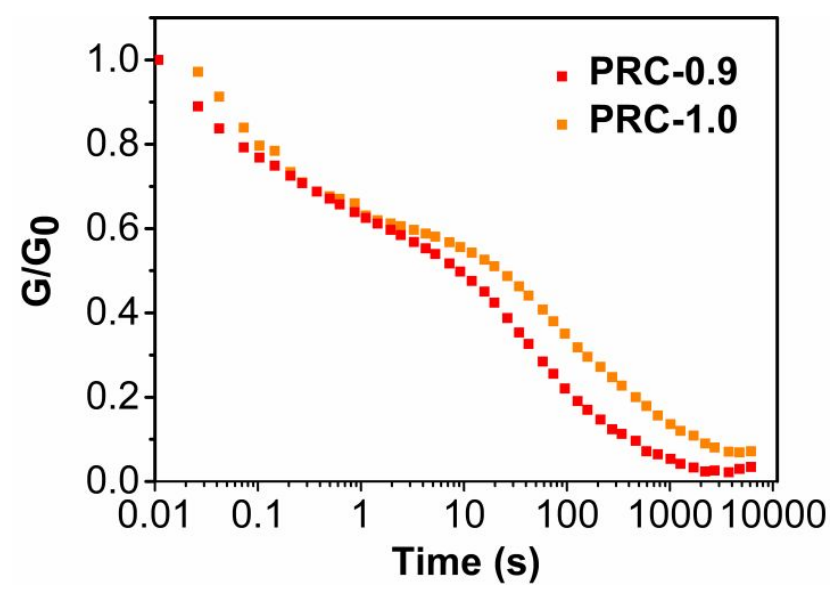

Figure S13. Stress relaxation of PRCs at $190{ }^{\circ} \mathrm{C}$ by rheology.

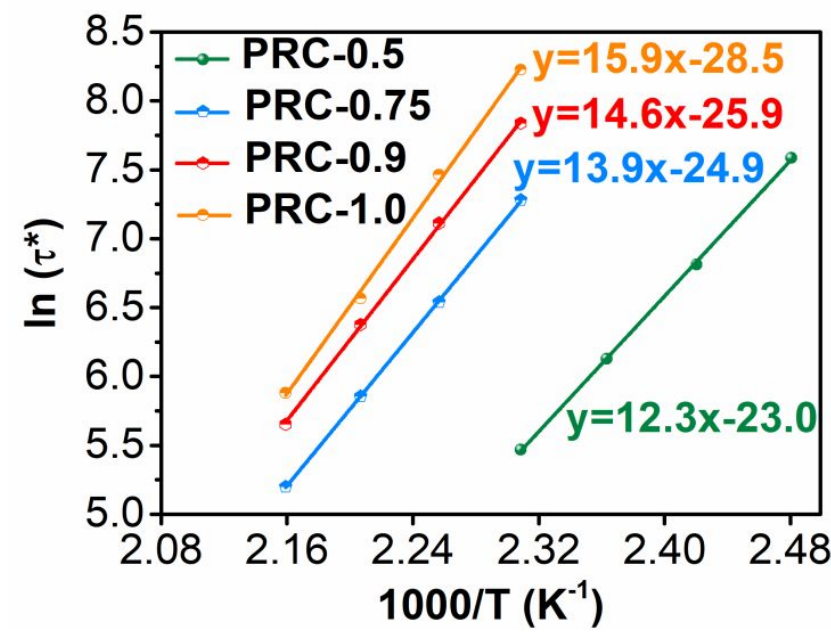

Figure S14. Arrhenius analysis of the characteristic relaxation time $\tau^{*}$ versus $1000 /$ T for PRCs. 
Supplementary note: Activation energies $\left(E_{a}\right)$ and Topology-freezing temperatures $\left(T_{v}\right)$ were determined using the methodology reported in literature. The characteristic relaxation times $\left(\tau^{*} \mathrm{~s}\right)$ were fit to the Arrhenius law in supplementary equation $1^{1}$.

\section{Supplementary equation 1: $\quad \tau^{*}(T)=\tau_{0}{ }^{*} e^{E_{d} / R T}$}

(R: universal gas constant; $8.314 \mathrm{~J} \mathrm{~K}^{-1} \mathrm{~mol}^{-1}$ )

Supplementary equation 2: $\quad \ln \tau^{*}(T)=\ln \tau_{0}{ }^{*}+E a / R T$

$T_{v}$ is the temperature at which the relaxation time $\tau^{*}$ is $10^{6} \mathrm{~s}$ according to the same method from Capelot et al ${ }^{2}$.

The measured values of characteristic relaxation times $\left(\tau^{*} \mathrm{~s}\right)$ were plotted versus $1000 / \mathrm{T}$.

$$
\begin{array}{ccc}
\text { PRC-0.5: } \mathrm{y}=12.3 \mathrm{x}-23.0 & E_{a}=102 \mathrm{~kJ} \mathrm{~mol}^{-1} & T_{v}=61^{\circ} \mathrm{C} \\
\text { PRC-0.75: } \mathrm{y}=13.9 \mathrm{x}-24.9 & E_{a}=115 \mathrm{~kJ} \mathrm{~mol}^{-1} & T_{v}=86^{\circ} \mathrm{C} \\
\text { PRC-0.9: } \mathrm{y}=14.6 \mathrm{x}-25.9 & E_{a}=121 \mathrm{~kJ} \mathrm{~mol}^{-1} & T_{v}=95^{\circ} \mathrm{C} \\
\text { PRC-1.0: } \mathrm{y}=15.9 \mathrm{x}-28.5 & E_{a}=132 \mathrm{~kJ} \mathrm{~mol}^{-1} & T_{v}=103{ }^{\circ} \mathrm{C}
\end{array}
$$

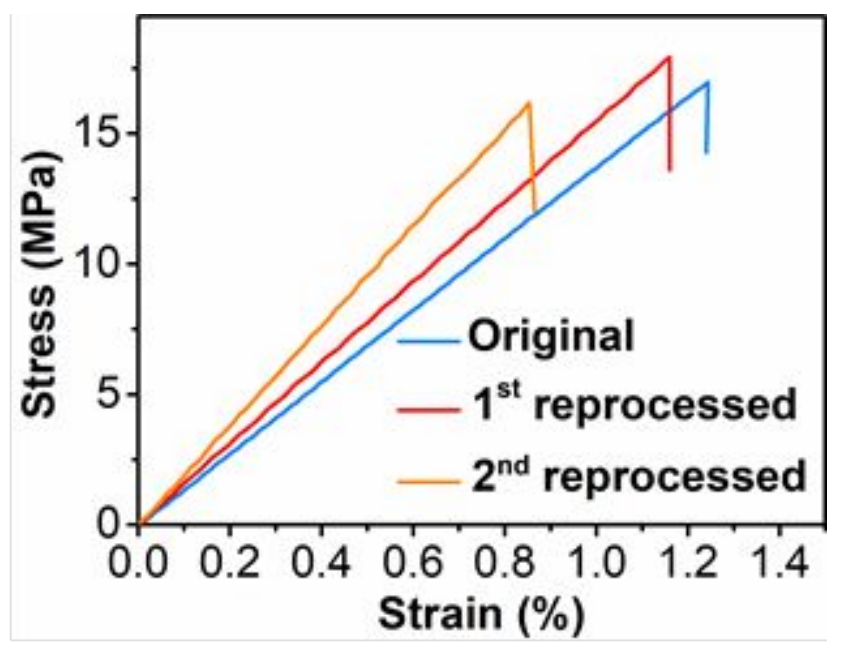

Figure S15. Representative tensile stress-strain curves of original and reprocessed PRC-0.5. 


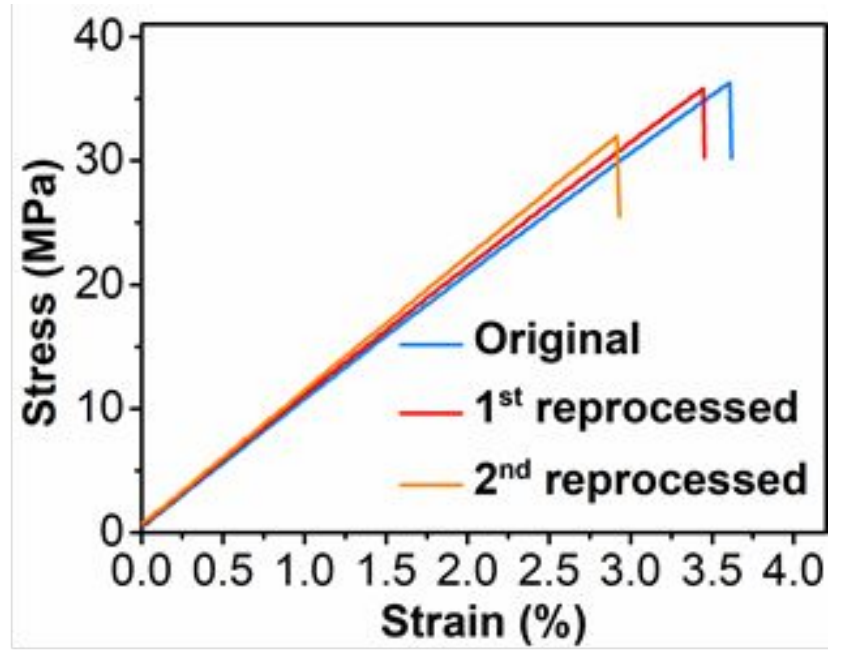

Figure S16. Representative tensile stress-strain curves of original and reprocessed PRC-0.75.

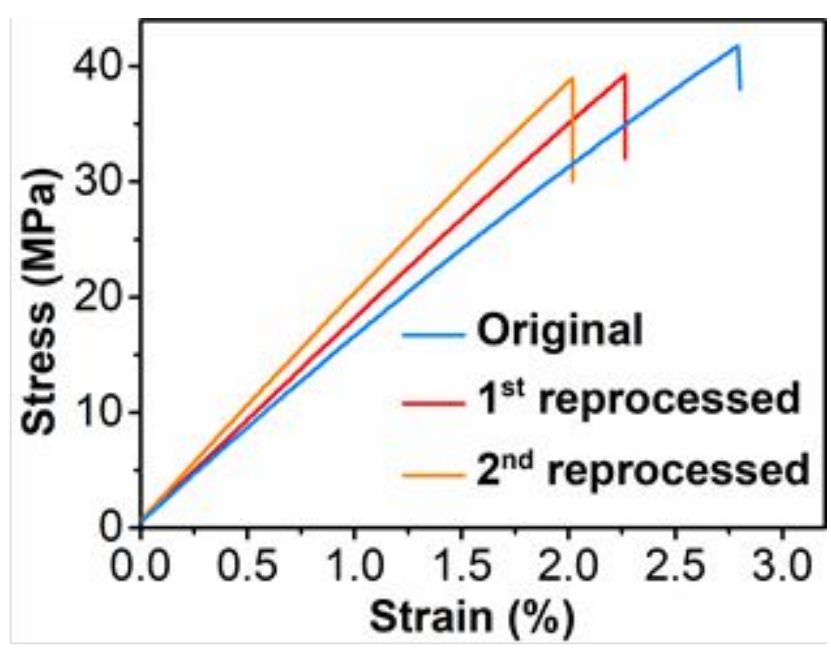

Figure S17. Representative tensile stress-strain curves of original and reprocessed PRC-1.0. 


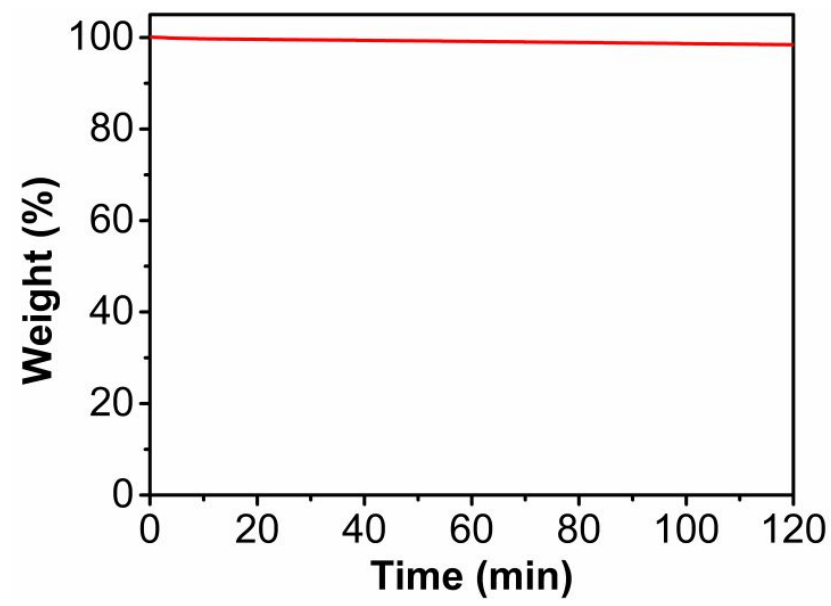

Figure S18. Isothermal TGA curve of PRC-0.9 at $170{ }^{\circ} \mathrm{C}$ for $2 \mathrm{~h}$.

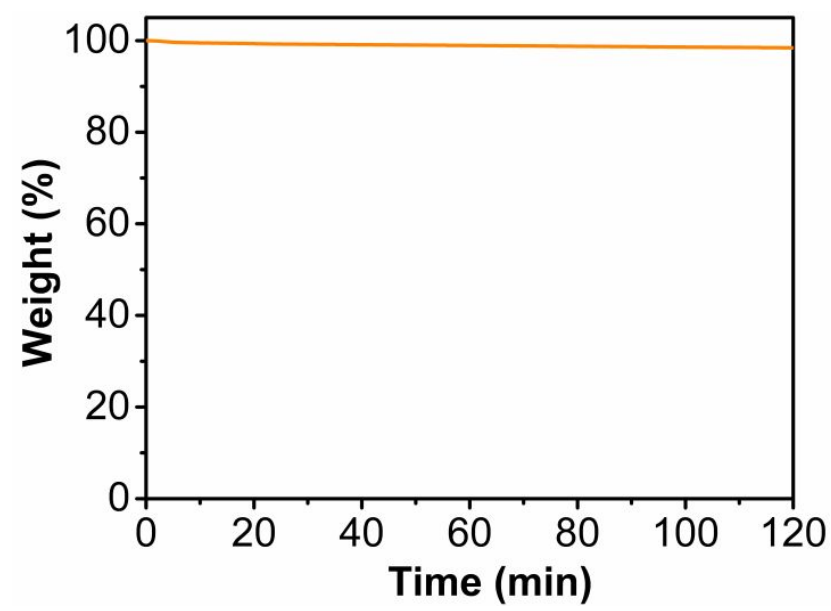

Figure S19. Isothermal TGA curve of PRC-1.0 at $170{ }^{\circ} \mathrm{C}$ for $2 \mathrm{~h}$. 


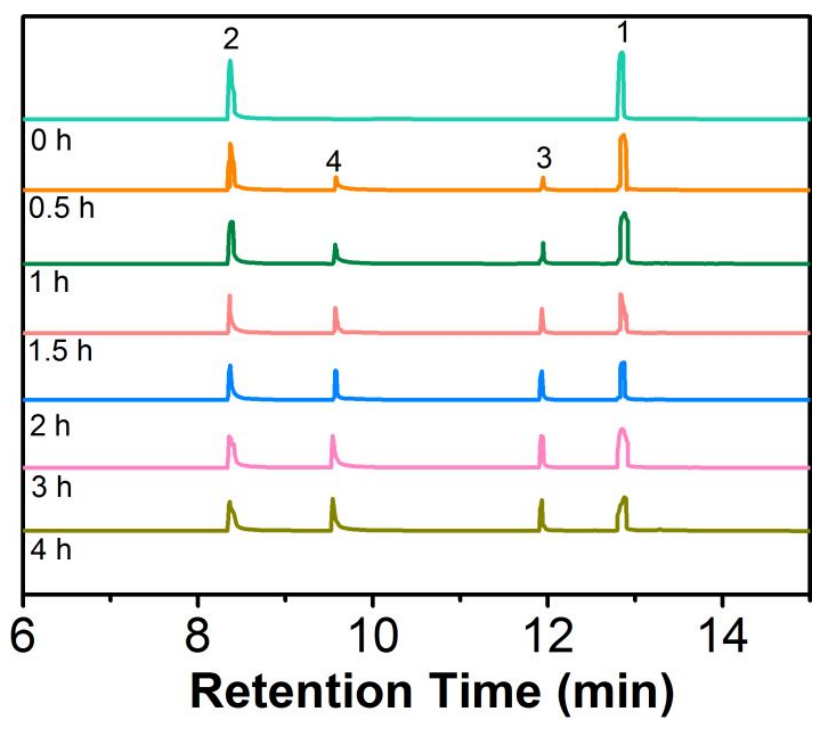

Figure S20. Gas chromatograms of transacetalization between model asymmetric acetal $\mathbf{1}$ and phenol $\mathbf{2}$ at $150{ }^{\circ} \mathrm{C}$ for different time.

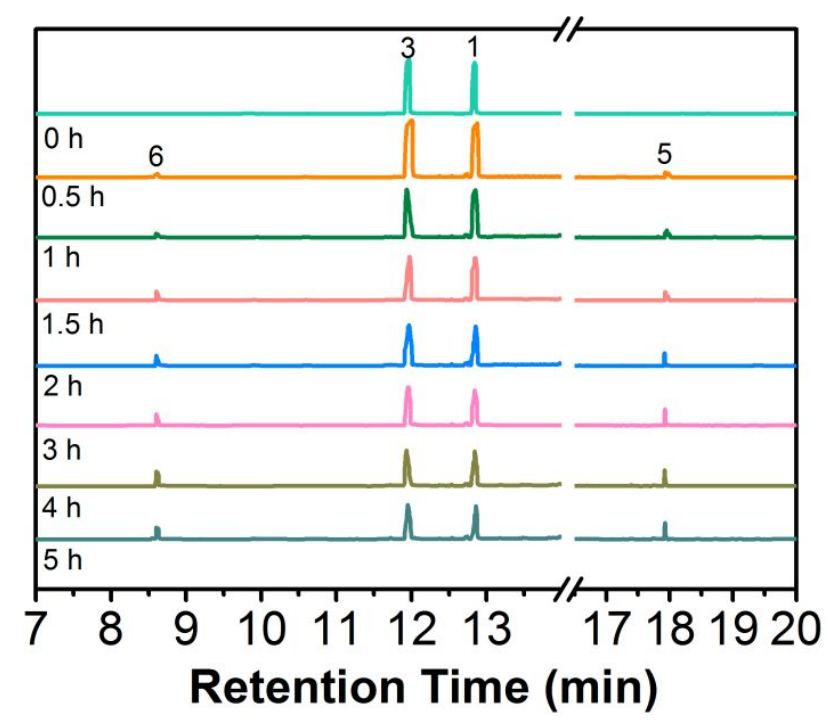

Figure S21. Gas chromatograms of metathesis between model asymmetric acetals $\mathbf{1}$ and $\mathbf{3}$ at $150{ }^{\circ} \mathrm{C}$ for different time. 


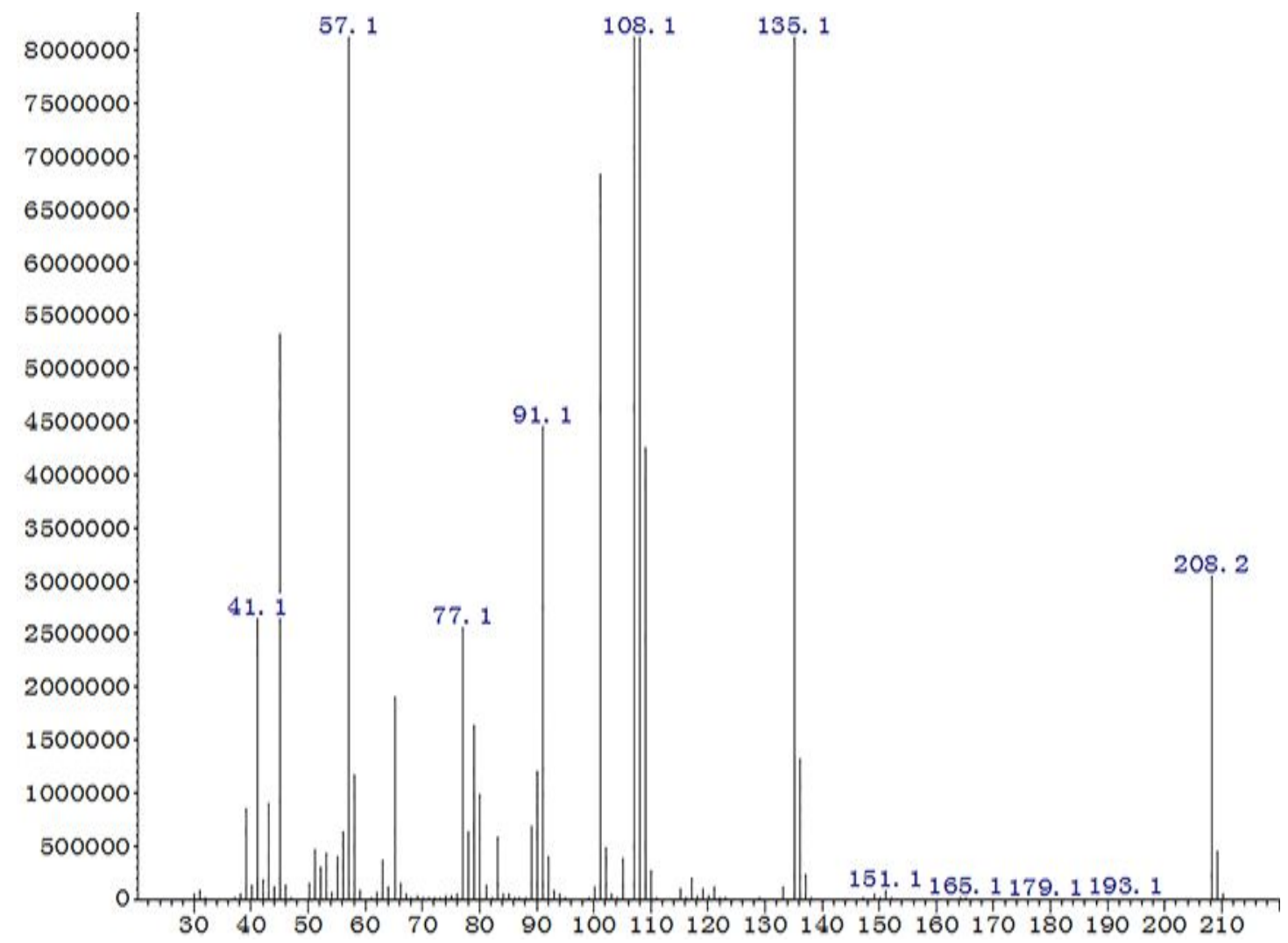

Figure S22. Mass spectrum of model asymmetric acetal 1 separated by gas chromatograph.

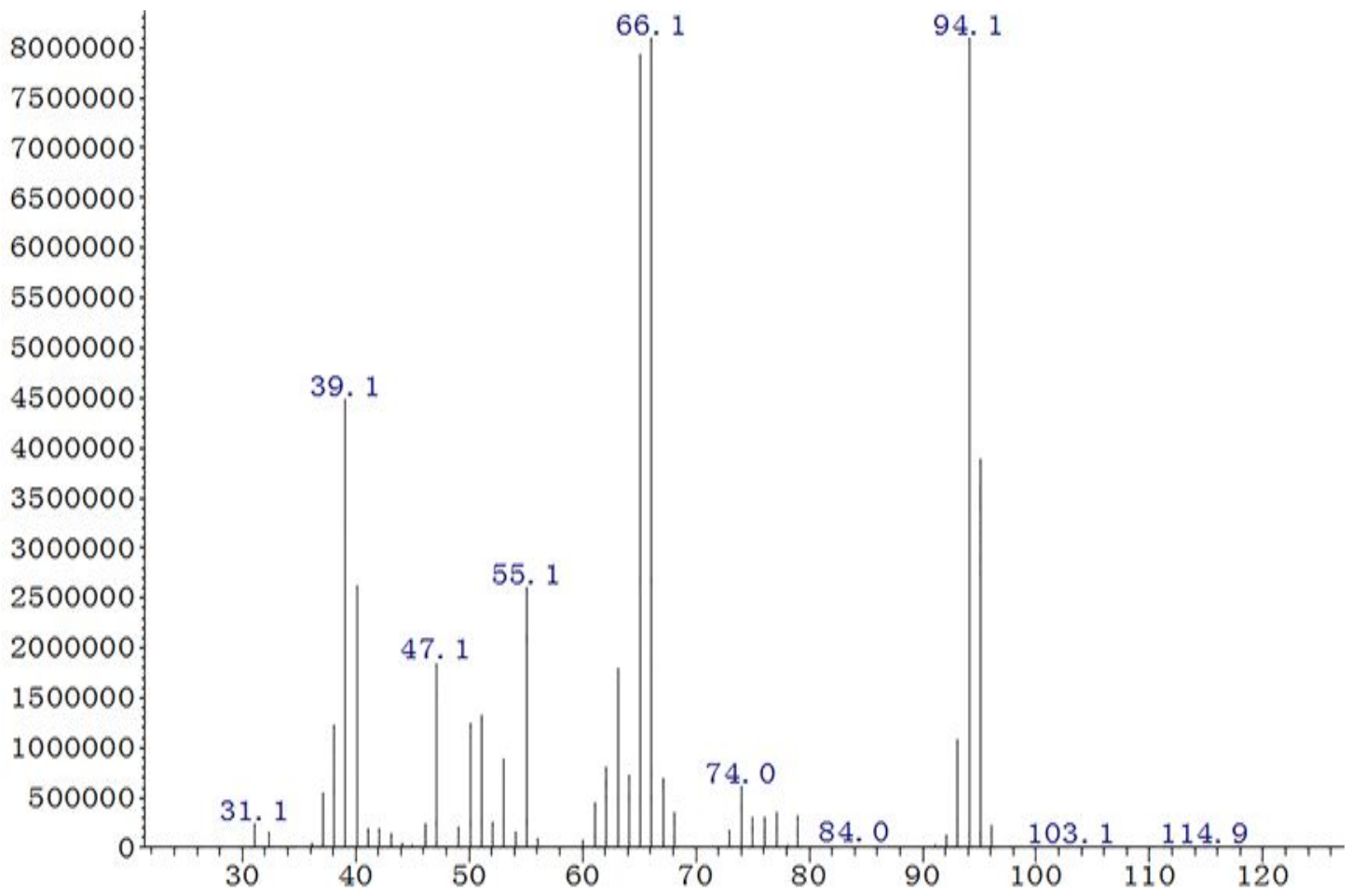

Figure S23. Mass spectrum of model phenol 2 separated by gas chromatograph. 


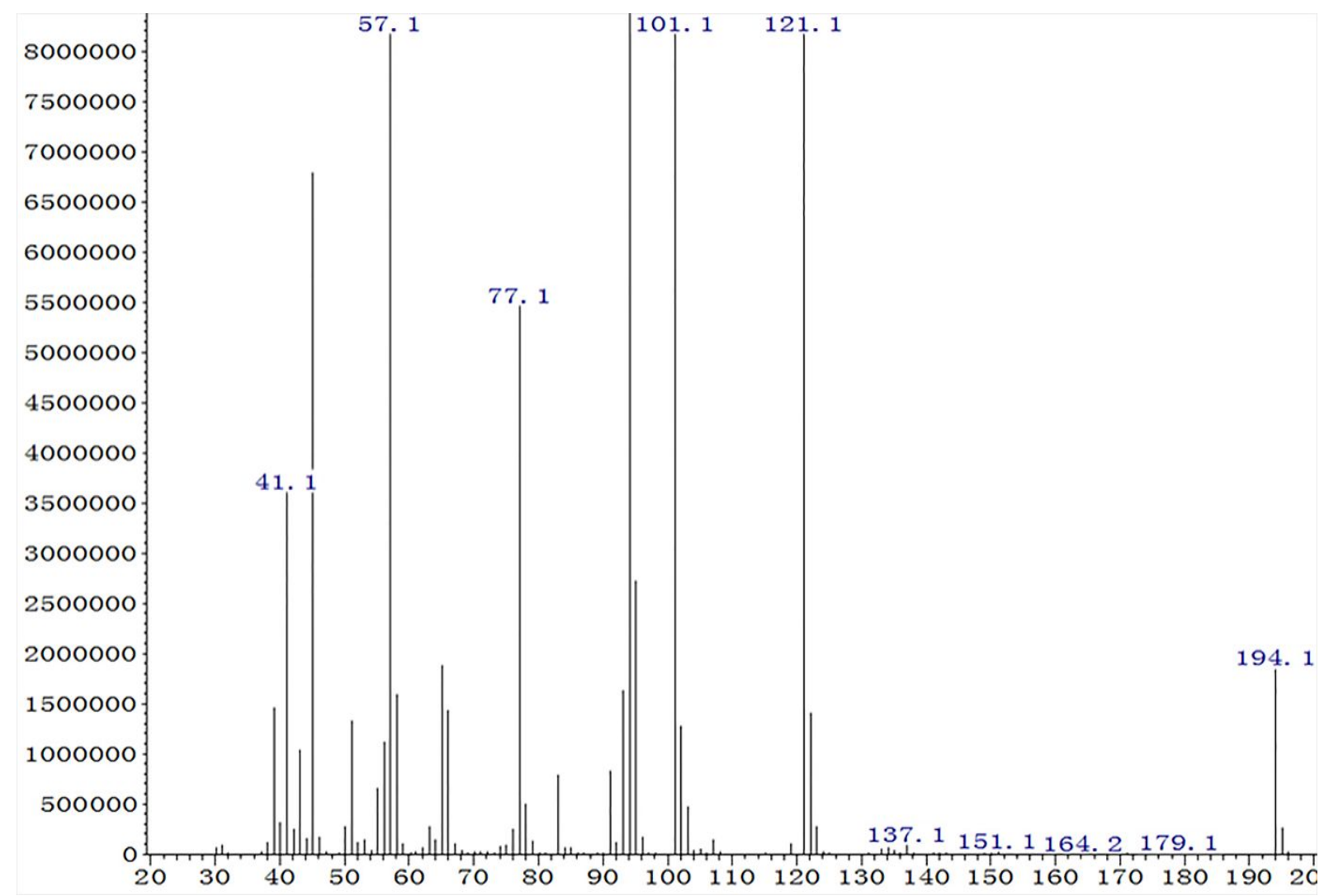

Figure S24. Mass spectrum of model asymmetric acetal 3 separated by gas chromatograph.

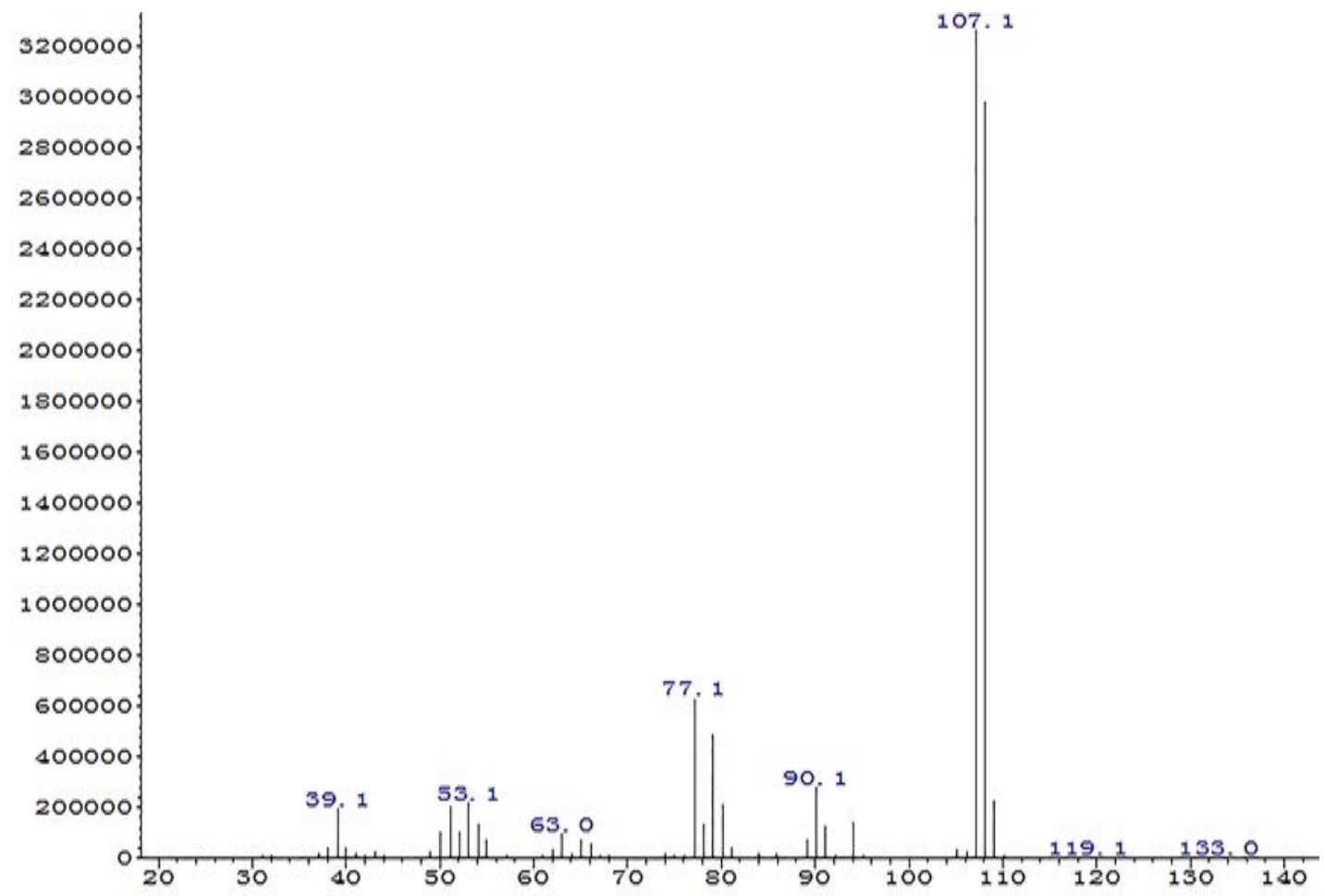

Figure S25. Mass spectrum of model phenol 4 separated by gas chromatograph. 


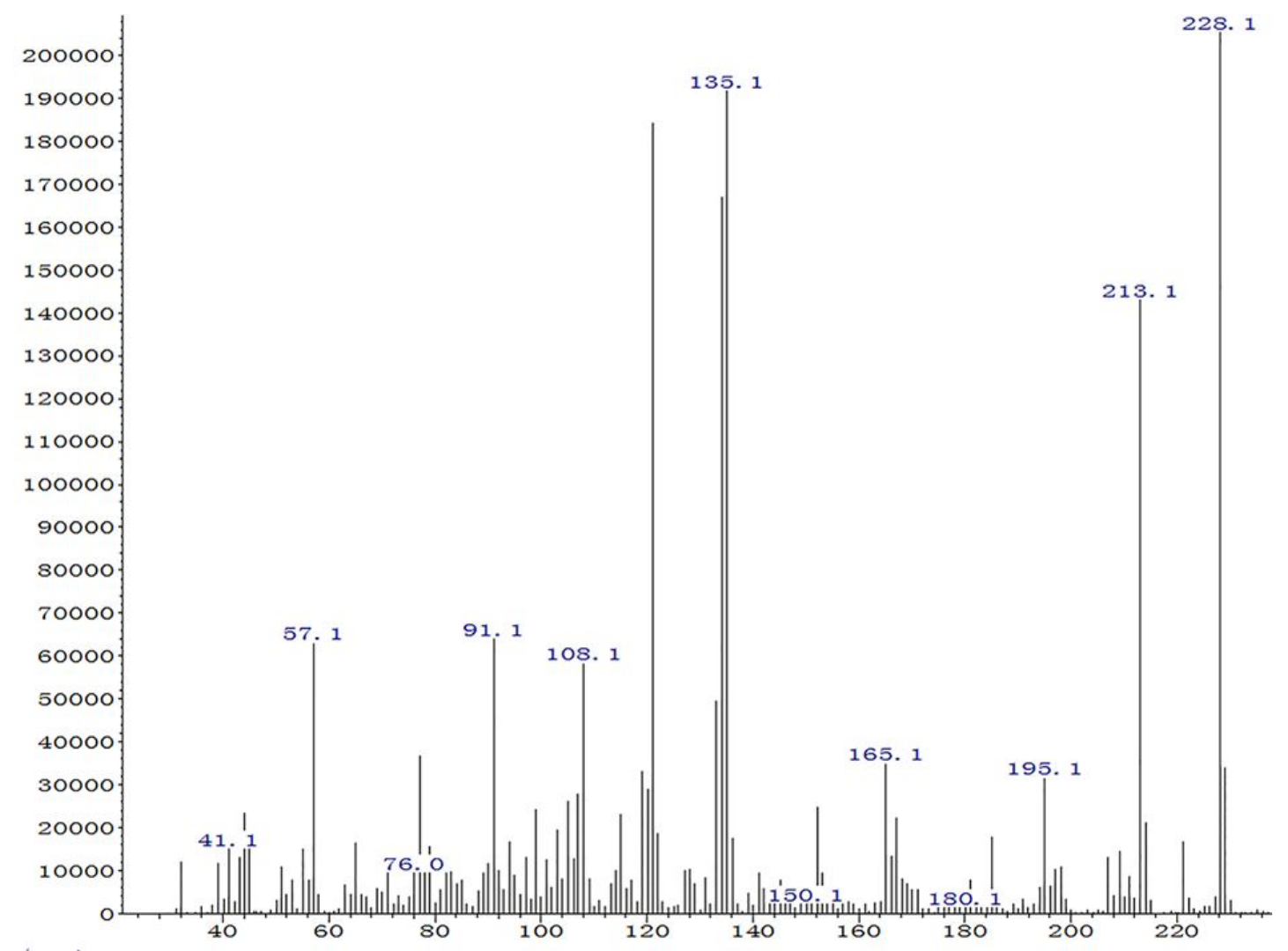

Figure S26. Mass spectrum of model asymmetric acetal $\mathbf{5}$ separated by gas chromatograph.

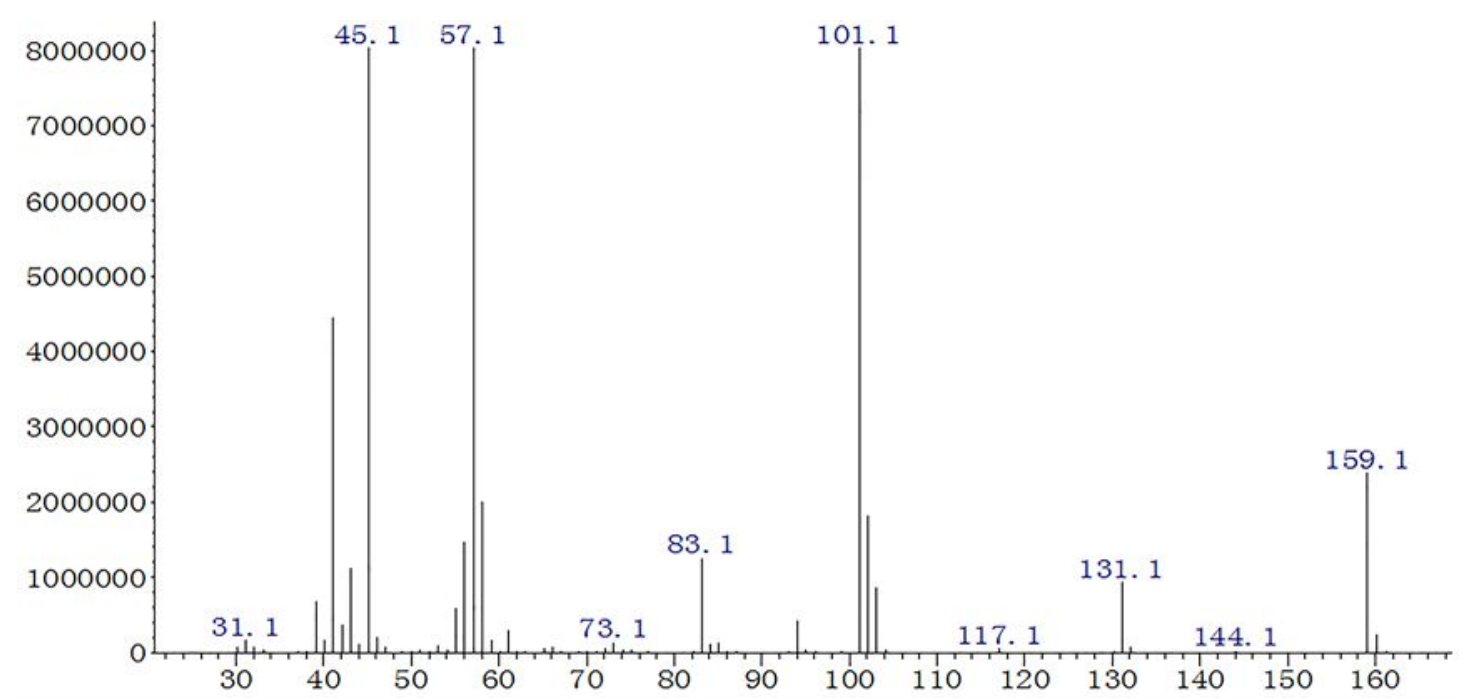

Figure S27. Mass spectrum of model asymmetric acetal $\mathbf{6}$ separated by gas chromatograph. 


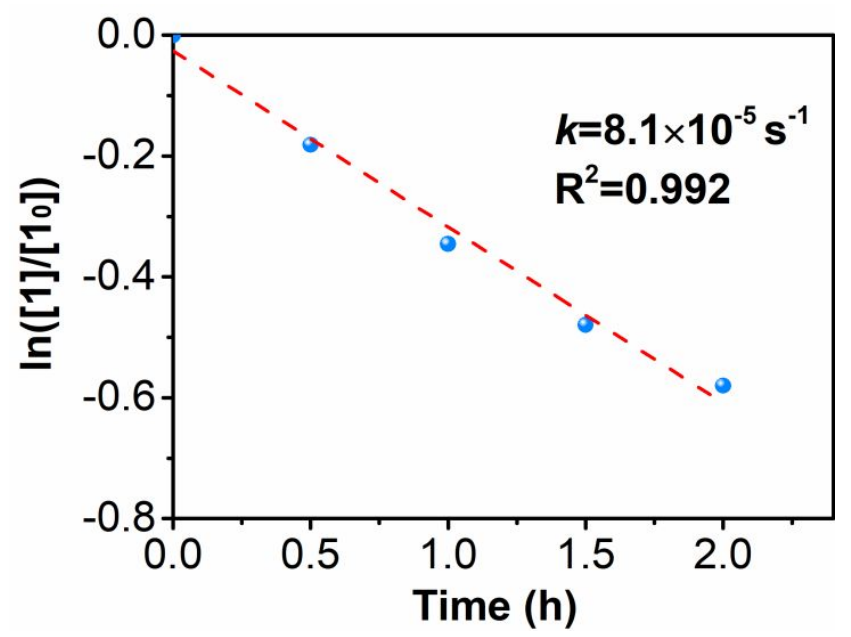

Figure S28. Pseudo-first order kinetics of transacetalization.

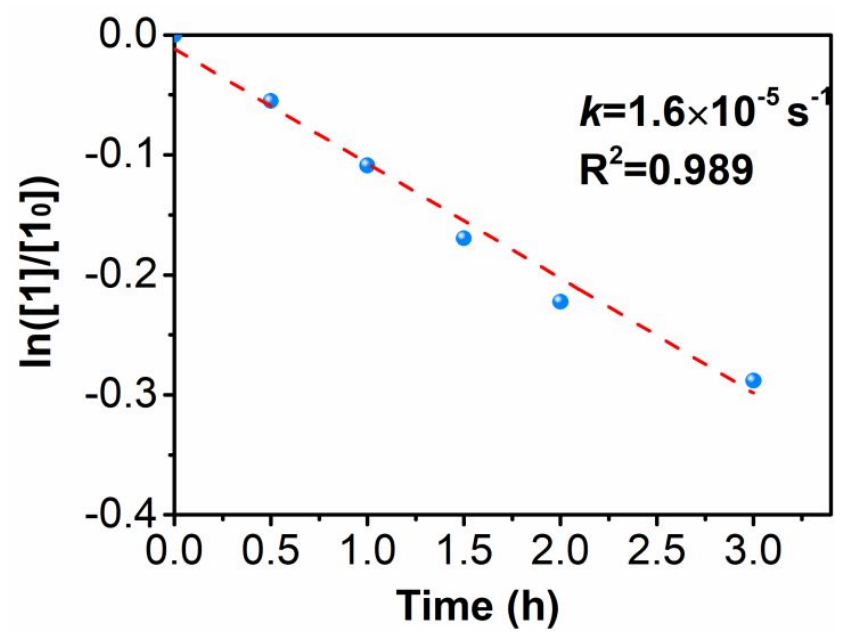

Figure S29. Pseudo-first order kinetics of metathesis. 

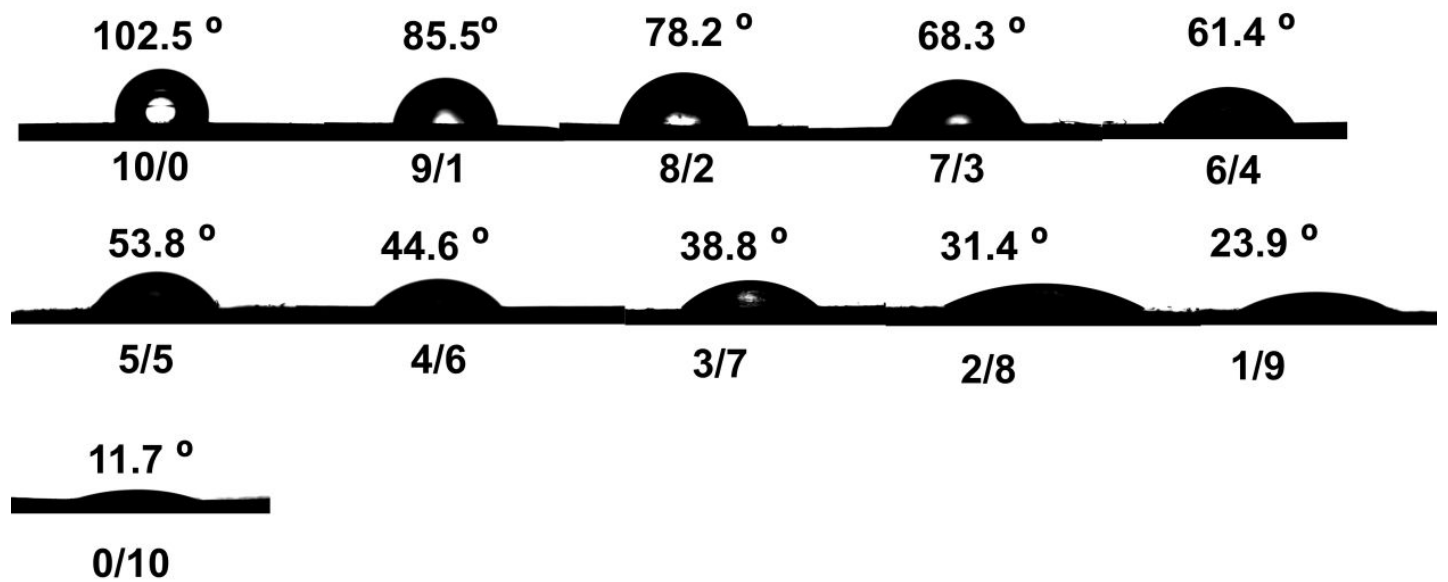

Figure S30. Contact angle of different solvents (water/acetone (v/v)) on PRC-0.9. 


\section{Supplementary Tables}

Table S1. The kinetic parameters of the different curing systems.

\begin{tabular}{cccccccc}
\hline \multirow{2}{*}{ sample } & \multicolumn{3}{c}{$T_{p}\left({ }^{\circ} \mathrm{C}\right)$ at different heating rate } & & \multicolumn{2}{c}{$E_{a}\left(\mathrm{~kJ} \mathrm{~mol}^{-1}\right)$} \\
\cline { 2 - 4 } \cline { 7 - 8 } & $5 \mathrm{~K} \mathrm{~min}^{-1}$ & $10 \mathrm{~K} \mathrm{~min}^{-1}$ & $15 \mathrm{~K} \mathrm{~min}^{-1}$ & $20 \mathrm{~K} \mathrm{~min}^{-1}$ & & Kissinger & Ozawa \\
\hline PRC-0.5 & 116.2 & 129.9 & 136.6 & 142.4 & & 64.7 & 67.9 \\
PRC-0.75 & 138.9 & 149.4 & 156.7 & 161.9 & & 82.7 & 85.3 \\
PRC-0.9 & 133.8 & 146.3 & 153.9 & 160.9 & & 69.0 & 72.2 \\
PRC-1.0 & 131.5 & 145.0 & 152.6 & 159.6 & & 65.5 & 68.9 \\
\hline
\end{tabular}

Table S2. Gel contents of PRCs in THF.

\begin{tabular}{cc}
\hline Samples & Gel content $(\%)$ \\
\hline PRC- 0.5 & $83.7 \pm 1.6$ \\
PRC-0.75 & $89.8 \pm 1.27$ \\
PRC-0.9 & $99.7 \pm 0.53$ \\
PRC- 1.0 & $99.9 \pm 0.10$ \\
\hline
\end{tabular}


Table S3. Swelling ratio of PRC in different solvent at $50^{\circ} \mathrm{C}$.

\begin{tabular}{|c|c|c|}
\hline Solvent & Samples & Swelling ratio $(\%)$ \\
\hline \multirow{4}{*}{ Acetone } & PRC-0.5 & $69.22 \pm 3.97$ \\
\hline & PRC- 0.75 & $60.05 \pm 3.16$ \\
\hline & PRC-0.9 & $50.58 \pm 2.67$ \\
\hline & PRC-1.0 & $38.18 \pm 2.37$ \\
\hline \multirow{4}{*}{ THF } & PRC- 0.5 & $62.13 \pm 3.57$ \\
\hline & PRC-0.75 & $53.71 \pm 3.03$ \\
\hline & PRC-0.9 & $41.71 \pm 2.17$ \\
\hline & PRC-1.0 & $36.18 \pm 2.59$ \\
\hline \multirow{4}{*}{ Methanol } & PRC-0.5 & $30.77 \pm 2.47$ \\
\hline & PRC-0.75 & $22.66 \pm 2.56$ \\
\hline & PRC-0.9 & $18.56 \pm 1.31$ \\
\hline & PRC-1.0 & $15.53 \pm 1.28$ \\
\hline \multirow{4}{*}{ Ethanol } & PRC-0.5 & $28.73 \pm 2.32$ \\
\hline & PRC-0.75 & $21.62 \pm 2.12$ \\
\hline & PRC-0.9 & $17.66 \pm 0.87$ \\
\hline & PRC-1.0 & $13.80 \pm 1.40$ \\
\hline \multirow{4}{*}{ DMF } & PRC-0.5 & $22.68 \pm 1.12$ \\
\hline & PRC-0.75 & $17.02 \pm 1.56$ \\
\hline & PRC-0.9 & $15.91 \pm 0.70$ \\
\hline & PRC-1.0 & $10.02 \pm 0.27$ \\
\hline \multirow{4}{*}{ Water } & PRC-0.5 & $3.19 \pm 0.25$ \\
\hline & PRC-0.75 & $2.31 \pm 0.13$ \\
\hline & PRC-0.9 & $1.48 \pm 0.20$ \\
\hline & PRC-1.0 & $0.95 \pm 0.10$ \\
\hline
\end{tabular}


Table S4. Mechanical properties of the original and reprocessed PRCs.

\begin{tabular}{|c|c|c|c|c|}
\hline Sample & & $\begin{array}{l}\text { Tensile modulus } \\
\qquad(\mathrm{MPa})\end{array}$ & $\begin{array}{c}\text { Tensile strength } \\
\text { (MPa) }\end{array}$ & $\begin{array}{c}\text { Elongation at } \\
\text { break }(\%)\end{array}$ \\
\hline \multirow{3}{*}{ PRC-0.5 } & original & $1843 \pm 59$ & $17.3 \pm 2.5$ & $1.3 \pm 0.3$ \\
\hline & $1^{\text {st }}$ reprocessed & $1984 \pm 78$ & $17.9 \pm 2.1$ & $1.2 \pm 0.5$ \\
\hline & $2^{\text {rd }}$ reprocessed & $2052 \pm 61$ & $16.2 \pm 1.6$ & $0.9 \pm 0.4$ \\
\hline \multirow{3}{*}{ PRC-0.75 } & original & $2477 \pm 66$ & $36.2 \pm 3.8$ & $3.6 \pm 1.1$ \\
\hline & $1^{\text {st }}$ reprocessed & $2543 \pm 72$ & $35.7 \pm 1.9$ & $3.4 \pm 0.9$ \\
\hline & $2^{\text {rd }}$ reprocessed & $2653 \pm 64$ & $32.0 \pm 3.6$ & $2.9 \pm 0.7$ \\
\hline \multirow{3}{*}{ PRC-0.9 } & original & $2766 \pm 103$ & $45.8 \pm 2.9$ & $2.4 \pm 0.6$ \\
\hline & $1^{\text {st }}$ reprocessed & $2806 \pm 110$ & $44.3 \pm 3.5$ & $2.2 \pm 0.5$ \\
\hline & $2 r^{d}$ reprocessed & $2936 \pm 123$ & $40.1 \pm 2.8$ & $1.7 \pm 0.4$ \\
\hline \multirow{3}{*}{ PRC-1.0 } & original & $2634 \pm 87$ & $41.9 \pm 3.1$ & $2.8 \pm 0.8$ \\
\hline & $1^{\text {st }}$ reprocessed & $2759 \pm 93$ & $39.2 \pm 4.3$ & $2.3 \pm 1.2$ \\
\hline & $2 r^{d}$ reprocessed & $2988 \pm 101$ & $38.9 \pm 4.7$ & $2.0 \pm 0.8$ \\
\hline
\end{tabular}


Table S5. Contact angle of different solvents on the surface of PRC-0.9.

\begin{tabular}{cc}
\hline $\begin{array}{c}\text { Water/Acetone } \\
(\mathrm{v} / \mathrm{v})\end{array}$ & Contact Angle $\left(^{\circ}\right)$ \\
\hline $10 / 0$ & $102.5 \pm 4.5$ \\
$9 / 1$ & $85.5 \pm 4.2$ \\
$8 / 2$ & $78.2 \pm 4.1$ \\
$7 / 3$ & $68.3 \pm 3.7$ \\
$4 / 6$ & $61.4 \pm 2.2$ \\
$5 / 5$ & $53.8 \pm 4.9$ \\
$6 / 4$ & $44.6 \pm 2.6$ \\
$3 / 7$ & $38.8 \pm 3.2$ \\
$2 / 8$ & $31.4 \pm 3.1$ \\
$1 / 9$ & $23.9 \pm 2.9$ \\
$0 / 10$ & $11.7 \pm 1.5$ \\
\hline
\end{tabular}


Table S6. Degradation rate of PRC-0.9 at different conditions.

\begin{tabular}{|c|c|c|c|c|c|}
\hline Main sovlent & $\begin{array}{l}\text { Water/Main solvent } \\
\quad(v / v)\end{array}$ & $\begin{array}{c}\text { Temperature } \\
\left({ }^{\circ} \mathrm{C}\right) \\
\end{array}$ & Acid & $\begin{array}{l}\text { Acid concentration } \\
\left.(\mathrm{mol} \mathrm{L})^{-1}\right)\end{array}$ & $\begin{array}{c}\text { Degradation Rate } \\
\left(\mathrm{mg} \mathrm{mL} \mathrm{h}{ }^{-1}\right)\end{array}$ \\
\hline Acetone & $1 / 9$ & 50 & $\mathrm{HCl}$ & 0.1 & $19.40 \pm 0.82$ \\
\hline Acetone & $2 / 8$ & 50 & $\mathrm{HCl}$ & 0.1 & $17.73 \pm 0.68$ \\
\hline Acetone & $3 / 7$ & 50 & $\mathrm{HCl}$ & 0.1 & $8.58 \pm 0.33$ \\
\hline Acetone & $4 / 6$ & 50 & $\mathrm{HCl}$ & 0.1 & $3.58 \pm 0.26$ \\
\hline Acetone & $5 / 5$ & 50 & $\mathrm{HCl}$ & 0.1 & $1.68 \pm 0.17$ \\
\hline Acetone & $6 / 4$ & 50 & $\mathrm{HCl}$ & 0.1 & $1.55 \pm 0.12$ \\
\hline Acetone & $7 / 3$ & 50 & $\mathrm{HCl}$ & 0.1 & $0.62 \pm 0.13$ \\
\hline Acetone & $8 / 2$ & 50 & $\mathrm{HCl}$ & 0.1 & $0.47 \pm 0.12$ \\
\hline Acetone & $9 / 1$ & 50 & $\mathrm{HCl}$ & 0.1 & $0.15 \pm 0.06$ \\
\hline Acetone & $1 / 9$ & 25 & $\mathrm{HCl}$ & 0.1 & $0.84 \pm 0.13$ \\
\hline Acetone & $1 / 9$ & 50 & $\mathrm{HCl}$ & 0.01 & $5.44 \pm 0.21$ \\
\hline Acetone & $1 / 9$ & 50 & $\mathrm{HCl}$ & 0.05 & $10.37 \pm 0.42$ \\
\hline Acetone & $1 / 9$ & 50 & $\mathrm{HCl}$ & 0.5 & $26.6 \pm 0.93$ \\
\hline Acetone & $1 / 9$ & 50 & $\mathrm{HCl}$ & 1 & $30.20 \pm 1.05$ \\
\hline Acetone & $1 / 9$ & 50 & $\mathrm{H}_{2} \mathrm{SO}_{4}$ & 0.1 & $23.97 \pm 0.42$ \\
\hline Acetone & $1 / 9$ & 50 & $\mathrm{H}_{3} \mathrm{PO}_{4}$ & 0.1 & $0.84 \pm 0.01$ \\
\hline Acetone & $1 / 9$ & 50 & $\mathrm{CH}_{3} \mathrm{COOH}$ & 0.1 & - \\
\hline THF & $1 / 9$ & 50 & $\mathrm{HCl}$ & 0.1 & $17.03 \pm 0.35$ \\
\hline Methanol & $1 / 9$ & 50 & $\mathrm{HCl}$ & 0.1 & $3.62 \pm 0.10$ \\
\hline Ethanol & $1 / 9$ & 50 & $\mathrm{HCl}$ & 0.1 & $1.83 \pm 0.10$ \\
\hline DMF & $1 / 9$ & 50 & $\mathrm{HCl}$ & 0.1 & $0.82 \pm 0.01$ \\
\hline no & $10 / 0$ & 50 & $\mathrm{HCl}$ & 0.1 & - \\
\hline
\end{tabular}


Table S7. Degradation rate of PRC- 0.9 at different conditions at $50{ }^{\circ} \mathrm{C}$ for $48 \mathrm{~h}$.

\begin{tabular}{cc}
\hline Solution & Weight Loss $(\%)$ \\
\hline $0.1 \mathrm{M} \mathrm{HCl}$ aqueous solution & $2.9 \pm 0.68$ \\
Water & $6.4 \pm 1.2$ \\
Water/Acetone $(\mathrm{v} / \mathrm{v}=1 / 9)$ & $0.27 \pm 0.05$ \\
\end{tabular}

\section{REFERENCES}

(1) Denissen, W.; Winne, J. M.; Du Prez, F. E. Chem. Sci. 2016, 7, 30-38.

(2) Capelot, M.; Unterlass, M. M.; Tournilhac, F.; Leibler, L. ACS Macro Lett. 2012, 1, 789-792. 